\title{
Gallbladder Polyp; When Surgical Treatment is Necessary?
}

\author{
İsmail Aydın ${ }^{1}$, Selçuk Göktaş² \\ ${ }^{1}$ Giresun University Faculty of Medicine, The Department of General Surgery, Giresun,Turkey \\ ${ }^{2}$ Bulancak State Hospital, General Surgery Clinic, Giresun,Turkey
}

Received: 24 August 2020, Accepted: 13 December 2020, Published online: 31 December 2020

(C) Ordu University Institute of Health Sciences, Turkey, 2020

\begin{abstract}
Objective: Gallbladder polyps (GBP) are the lesions that are originated from the mucosa of the gallbladder and reach out to the lumen. The biological behaviours of GBP have still been uncertain, their follow-ups and treatments are controversial as they carry the risk of malignancy. Our aim is to present the results of patients who have been operated on with the diagnosis of GBP in this study.

Methods: This retrospective study was conducted at Department of General Surgery, Giresun University. Patients who underwent surgery for GBP were included between January 2015 and December 2019. The age, gender, symptoms, ultrasonography findings (numbers polyps, types and presence of polyp and stone), surgery method (open and laparoscopic), and histopathological examination results were analysed.

Results: A total of 1486 cholecystectomies, including 1388 laparoscopic cholecystectomy and 98 open cholecystectomies, were performed. One-hundred thirty-two (8.8\%) of these patients were operated with the diagnosis of the GBP. One-hundred thirty of them were operated with the laparoscopic method and two of them with the open surgical method. Overall, fourty two $(31.8 \%)$ of the patients were male and $90(68.2 \%)$ were female. The average age was 48.7 . One-hundred one $(76.5 \%)$ patients were clinically symptomatic. Gallstones were analysed in 35 (26.5\%) patients. Multipl polyps were detected in 34 (25.8\%) patients. There were no polyps determined in $47(35.6 \%)$ patients the histopathological examination. The most common polyp type was cholesterol polyps (\%64.8). Cancer histopathology was not found in any patient.

Conclusion: It is difficult to differentiate premalign and/or malignant lesions of the gallbladder from benign lesions. Therefore, we believe that surgical treatment is the correct approach for all GBP, which are symptomatic, accompanying stones, and that have a risk of malignancy ultrasonographically.
\end{abstract}

Key words: Gallbladder, polyp, cholecystectomy

Suggested Citation: Aydin I, Goktas S. Stopping ultrafiltration related blood pressure changes in hemodialysis patients. Middle Black Sea Journal of Health Science, 2020; 6(3):369-375.

\section{Address for correspondence/reprints:}

İsmail Aydın

Telephone number: +90 (532) 6365794

ORCID-ID 0000-0002-9177-6298

E-mail: drisoaydin78@gmail.com

DOI: $10.19127 / \mathrm{mbsjohs.784868}$

\section{Introduction}

Gallbladder polyps (GBP) are defined as lesions that are mostly benign nature and originated from gallbladder mucosa reach out to the lumen. They are found in $0.3-12 \%$ of healthy individuals (Chaet al., 2011). The true prevalence is unknown yet. They usually diagnosed as a result of radiologic examination performed for other reason and appear without any symptom. GBP are divided into two categories as pseudopolyp and true polyps. Pseudopolyps consist of cholesterol polyps/cholesterolosis, adenomyoma, inflammatory 
polyps, and hyperplastic polyps. All of them are benign lesions. True polyps are grouped as benign (adenoma), premalignant (dysplastic polyps), and malignant (adenocarcinoma) (Zielinski et al., 2009). The abdominal imaging techniques has caused a dramatic increase in the diagnosis of a polyp (Lin et al., 2008, Cairns et al., 2012, Marangoni et al., 2012, $\mathrm{Xu}$ et al., 2012). Adenomas constitute $10 \%$ of SKPs and are found in approximately $1 \%$ of cholecystectomy specimens (Sun et al., 2019). GBP is detected in 3-7\% of people who undergo ultrasonography (US) and at the rate of $2-12 \%$ in cholecystectomy specimens (Yuksel et al. 2016). The disease is observed more commonly in the age of forties and in women (Dinc et al., 2013). GBP mostly consist of cholesterol polyps. Even though it is considered to have no potential for malignancy, the US images of polyps are like the early stages of gallbladder cancer. The risk of malignancy development increases especially in the presence of polyps with adenomatous features (Dinc et al., 2013) over $10 \mathrm{~mm}$, and three or more polyps regardless of the diameter. It has been thought that gallbladder cancer occurs due to the malignant transformation of adenoma (adenoma- adenocarcinoma sequence) based on the diameter of polyp (Yuksel et al. 2016). Hence, the diagnosis and treatment of the polyp in the gallbladder become crucial in a way of detecting cancer in an early stage. In this study, the data of the patients, who were operated with the diagnosis of GBP were examined and the results were aimed to be discussed in the light of the literature.

\section{Methods}

The data of patients who were operated with the diagnosis of GBP between January 2015 and November 2019 at the Department of General Surgery of the Ministry of Health of Giresun University Prof. Dr. A. İlhan Özdemir Education and Research Hospital were analyzed through the hospital's electronic patient file archive. Patients whose preoperative examinations were performed in another hospital were not included in the study. All surgeries, radiological and pathological examinations were performed by specialist physicians in our hospital. The age, gender, presence of symptoms, US findings ( the number of polyps, the dimension of the polyp, the presence of stone), surgery method (open or laparoscopic) the results of histopathological examination of patients were included to study after obtaining the necessary permissions. The diagnosis of polyp at US was established according to the criterias of projection of lesion to the lumen, inability to form an acoustic shadow, and inability to be replaced. According to histopathological findings, cholesterol polyps/cholesterolosis, hyperplastic polyps and polyps with adenomyomatosis were evaluated as nonneoplastic polyps, adenomas benign neoplastic polyps, and adenocarcinomas were examined as neoplastic polyps. Histopathological results are assessed by pathologist in hospital.

\section{Statistical analysis}

Statistical analysis was performed using the SPSS 23.0 software (IBM Corporation, Armonk, NY, USA). For the data analysis, SPSS package program version 23.0 was used. The descriptive statistics were given to define and to explore properties of sample units in the research. The agreement among pathology and US decisions for determining polyp stone has been measure by using Cross tables and kappa statistics. Moreover, the significances of the Kappa statistics were tested by using statistical hypothesis test. P-value $<0.05$ was considered as statistically significant.

\section{Results}

A total of 1486 cholecystectomies, including 1388 laparoscopic cholecystectomy and 98 open cholecystectomy, were performed. One-hundred thirty two $(8.8 \%)$ of these patients were operated with the diagnosis of GBP. The cross-tabulation for these patients is given in Table 1. One-hundred thirty of them were operated with the laparoscopic method and two of them with the open surgical method. Overall, forty two $(31.8 \%)$ of the patients were male and 90 $(68.2 \%)$ of them were female. The average age was $48 \pm 13$ years. The polyp-stone coexistence was determined for $18.2 \%$ of patients in both US and histopathologically examination. Both US and pathology have found that there was no polyp-stone coexistence for $56.1 \%$ of patients. The results of US and histopathology were coherent for $74.3 \%$ of patients in total. The kappa coefficient obtained for the compliance of US and histopathology in determining the polyp-stone coexistence was 0,447 and it shows moderate concordance. It was also concluded that this coefficient indicates a nonignorable statistically significant compliance according to approximate significance value ( $\mathrm{p}$ value) (Table-1). 


\section{Gallbladder Polyp; When Surgical Treatment is Necessary?}

Table-1. US Polyp-Stone, Pathology Polyp Stone Cross-tabulation

\begin{tabular}{|c|c|c|c|c|c|}
\hline & & & \multicolumn{2}{|c|}{ Pathology Polyp Stone } & \multirow[b]{2}{*}{ Total } \\
\hline & & & Yes & No & \\
\hline \multirow[t]{6}{*}{ US Polyp-Stone } & Yes & $\mathrm{n}$ & 24 & 11 & 35 \\
\hline & & $\%$ of Total & 18.2 & 8.3 & 26.5 \\
\hline & No & $\mathrm{n}$ & 12 & 74 & 86 \\
\hline & & $\%$ of Total & 9.1 & 56.1 & 65.2 \\
\hline & Undefined & $\mathrm{n}$ & 4 & 7 & 11 \\
\hline & & $\%$ of Total & 3.0 & 5.3 & 8.3 \\
\hline \multirow[t]{2}{*}{ Total } & & $\mathrm{n}$ & 40 & 92 & 132 \\
\hline & & $\%$ of Total & 30.3 & 69.7 & 100.0 \\
\hline Kappa coefficient & & $\mathrm{P}=0.000$ & & & \\
\hline
\end{tabular}

The relationship between the number of polyps determined according to US and the number of polyps determined according to histopathology was investigated with the Kappa coefficient by creating a cross-tabulation (Table 2). While US was determining a single polyp for $23.5 \%$ of patients and multiple polyps for $12.1 \%$ of the patients, there is no polyp established as a result of histopathology. There whilst US was detecting a single polyp for $16.7 \%$ of patients and multiple polyps for $13.6 \%$ patients, the single polyp was determined as a result of the pathology of the same patients. While US was determining a single polyp in $8.3 \%$ of patients and multiple polyps in $25.8 \%$ of patients, the multiple polyps were identified in these patients in consequence of pathology. US was detecting a single polyp in $35.6 \%$ of patients whereas there is no polyp identified in the pathology results of the same patients. Kappa coefficient determining the compliance in the number of polyps between US and histopathology was obtained as 0.15 and showed a weak but statistically significant concordance since $\mathrm{p}<0.01$ (Table-2).

Table-2. Numbers of polyp US, Number of polyp Pathology Cross-tabulation

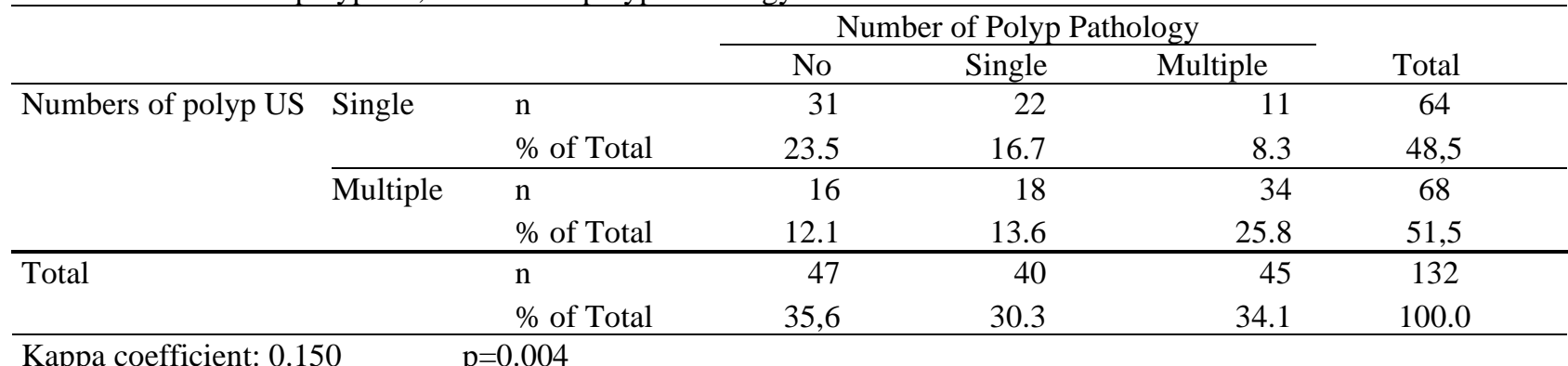

Table-3. Symptom, US Polyp-Stone Cross-tabulation

\begin{tabular}{|c|c|c|c|c|c|c|}
\hline & & & \multicolumn{3}{|c|}{ US Polyp-Stone } & \multirow[b]{2}{*}{ Total } \\
\hline & & & Yes & No & Undefined & \\
\hline \multirow[t]{4}{*}{ Symptom } & Yes & $\mathrm{n}$ & 25 & 66 & 10 & 101 \\
\hline & & $\%$ of Total & 18.9 & 50.0 & 7.6 & 76.5 \\
\hline & No & $\mathrm{nt}$ & 10 & 20 & 1 & 31 \\
\hline & & $\%$ of Total & 7.6 & 15.2 & 0.8 & 23.5 \\
\hline \multirow[t]{2}{*}{ Total } & & $\mathrm{nt}$ & 35 & 86 & 11 & 132 \\
\hline & & $\%$ of Total & 26.5 & 65.2 & 8.3 & 100.0 \\
\hline
\end{tabular}


One-hundred one $(76.5 \%)$ patients were clinically symptomatic. Gallstones were found in 35 (26.5\%) patients. Multiple polyps were present in $68(51.5 \%)$ patients (on pathological examination) and 34 (25.8\%) patients (on US examination). The Kappa coefficient obtained through the cross-tabulation (Table-3) which was created to identify the effect of presence or absence of symptoms for US to determine the polyp-stone coexistence shows that there is no statistically significant relation (Table-3).

Cholesterol polyps were the most frequently detected polyp types in this study. When all the operated patients were evaluated, this rate was found to be $64.4 \%$. Pseudopiloric metaplasia in company with polyp was detected in one patient, xanthogranulomatous cholecystitis without polyp in one patient, and adenomyosis was determined in three patients. Neoplastic polyp and invasive cancer were not identified in any patients.

\section{Discussion}

The biological behaviors of GBP have still been uncertain (Yildirim et al., 2005). There are studies that report the gender distribution is equal or more frequent in females. The rate of incidence is after the age of forties (Yildirim et al., 2005). The average age of the patients in our study was $48 \pm 13$ years and $68.2 \%$ of all patients were female.

Even though many of the polyps consist of benign cholesterol polyps, the risk of malignancy is still the biggest problem. Hence, the diagnosis, follow-up and treatment are crucial (Dincet al., 2013). It has been reported that the rate of incidental diagnosis of GBP is $7-20 \%$ and it is mostly asymptomatic. The most common symptom is right upper quadrant pain in symptomatic cases. In addition, hemobilia and obstructive jaundice have been indicated as symptoms (Yildirim et al., 2005). US examination is the most preferable tool in the diagnosis of GBP. In US, polyps are evaluated as lesions that are hyperechoic, are without posterior shadows, and cannot displace by position. The sensitivity of US for the GBP has been reported as between 32\% and 90\% in the literature (Yuksel et al. 2016). However, the most important restrictive factors are; dependency of US to the person, not being standardized and the factors that belong to the patient (obesity, accompanying gallstones etc.) affect the correct diagnosis (Yuksel et al. 2016). Although the sensitivity of conventional US is not high in the benign and malign differentiation of cases with GBP, the morphological appearance of the polyp can provide insight. Considering the studies, it has been reported that the imaging methods, except US, such as computerized tomography and endoscopic retrograde cholangiopancreatography have lower values for the diagnosis of polyps than US and have higher costs. Therefore, the most frequently used US examination has still been today (Yuksel et al. 2016). In our study, all diagnosis was established via US. After the histopathological examination polyps were detected in rate of $64.4 \%$ in patients with polyps that were determined through US in our study.

In a study with 194 patients that was conducted by Sun et al., it has been indicated that US was the most suitable diagnostic method for the diagnosis of the polyp and using computerized tomography (CT) was recommended for suspicious lesions (Sun et al., 2004). In a study that was conducted by Lou et al., it has been stated that $\mathrm{CT}$ was a sensitive and reliable technique in order to show the polyps of the gallbladder and the lesions up to $1.5 \mathrm{~mm}$ in length easily (Lou et al., 2004). Moreover, techniques such as endoscopic US (EUS), contrast EUS, and magnetic resonance imaging (MRI) are recommended in polyps with high-risk factors in terms of neoplastic features (Sun et al. 2019). In our study, the median of polyp diameters determined according to US was found five and the median of polyp diameters established according to pathology was found three. Therefore, it is concluded that the diameters that were identified according to US were higher.

The essential topic in patients with a polyp in the gallbladder is that how the follow-up and the treatment procedure will take place. Hereby, we have the risk of polyp malignancy. It has been reported in the studies that are conducted, the GBP are malignant at the rate of 3-8\% (Goetze et al., 2010). Establishing an early diagnosis becomes crucial when the poor prognosis of gallbladder cancers is considered. The segregation between benign and malign cannot be performed with any of the preoperative imaging techniques (Kwon et al., 2009). Folded gallbladder, mucosal residues or stones that are impacted to the wall may have a role in the false-positive rate in US examination. In our study, US was determining a polyp for $35.6 \%$ of the patients while any kind of polyp could not be identified as a result of histopathology for the same patients. We believe this circumstance has occurred due to the folded gallbladder, mucosal residues, stones impacted to the wall, missing the small polyps with thin stems with the gall flow during the pathological examination, and the person who performed US.

In a prospective study that was conducted by Shinkai et al., (1998) the diameter of lesion and echogenicity remained the same in $93 \%$ of the patients who were following-up due to the polyp in 
gallbladder. Furthermore, in a study that was done by Csendes et al., (2001) polyp diameters were constant for $50 \%$ of the patients while there was an increase detected in the polyp diameters for $25 \%$ of them for six years follow-up periods of 98 patients. The adenomatous polyp was detected in one patient among the patients who had an increase in polyp diameters and the rest of them were reported as cholesterol polyps (Dincet al., 2013). In the present study, due to the increase in the diameters and numbers of the polyp, the operation was decided for five patients in one year of follow-up and all of those patients were reported as cholesterol polyps.

Cholesterol polyps are the most common type of GBP. Histologically, they are formed by histiocytes including cholesterols covered by single-row epithelium. Their etiologies are not completely known. Directly storing the serum cholesterols in the gallbladder, aggregation of free sterols from gall or the transformation after cholesterol synthesis of the liver are the defended mechanisms in cholesterolysisetiology (Yildirim et al., 2005). True neoplastic polyps, on the other hand, show a broad spectrum and malignancy is more common in patients older than 50, in lesions that are single and larger than $10 \mathrm{~mm}$, with gallstones or show ultrasonographic rapid grow in a short period of time even the ones smaller than $10 \mathrm{~mm}$. The prevalence of neoplastic polyps has been stated as 5-10\% (Jones-Monahan et al., 2000). There were no neoplastic polyps detected in our study.

Atypia and dysplasia have been reported in various ratios in adenomas. The development of metaplasia in polyp has a higher rate especially in patients who have stones in the gallbladder. This rate has been stated as $48 \%$ particularly in adenomatosis polyps (Yildirim et al., 2005). Polyps including atypia and dysplasia have not been identified in our study.

The treatment algorithm of GBP has still been controversial. Shinkai et al. (1998) have recommended for cholesterol polyps which are above $10 \mathrm{~mm}$ to be operated with laparoscopic cholecystectomy which is a minimally invasive method. Csendes et al. (2001) have suggested to apply cholecystectomy for lesions with over $10 \mathrm{~mm}$ polyp dimension and to perform open cholecystectomy when the risk of malignancy is taken into consideration. Lee et al. (2004) have stated to perform frozen section examination and to treat with open cholecystectomy for polyps' cases with over 20 $\mathrm{mm}$ dimension. In our study, $98.5 \%$ of the patients were operated with laparoscopy and $1.5 \%$ of them with open cholecystectomy. The decision to open surgery arisen from previous abdominal surgery.
Some of the risk factors have been stated for the malign GBP in the literature. Polyp size over $10 \mathrm{~mm}$, patients older than the age of 50, being symptomatic, solitary and sessile polyp, accompanying gallstones are the risk factors that were identified (Sarkut et al., 2013).

The relationship between the symptoms and risk of malignancy is not clear.However, according to some research, there is a connection between the symptoms and malignancy (Sun et al. 2019) whereas some of them could not find any relationship (Albores-Saavedraet al., 2012). The mechanism underlying the malign transformation is unclear and is required more molecular research (Sun et al. 2019). The neoplastic polyp was not determined in our study. Additionally, the communications between the polyp and presence and/or absence of symptoms and between polyp-stone coexistence could not be formed.

Cholecystectomy is accepted in symptomatic cases regardless of the size. The surgical method should also be applied in cases that are not symptomatic but above the age of 50, that the polyp size is above $10 \mathrm{~mm}$, in sessile morphology, with accompanying gallstones since malignancy cannot be excluded in those cases. In other cases, six months follow-up with US is recommended. During this period, if an extension is detected in polyp sizes or symptoms are developed, surgical treatment becomes a current issue. If the polyp disappears in the US during follow-up, it is recommended to remove those cases from follow-up (Yildirim et al., 2005). Cholecystectomy should be taken into consideration in GBP that shows the intra-lesional bloodstream regardless of the size by virtue of high risk for neoplastic polyp (Sun et al. 2019). In our study, all of the patients had one or more of the risk factors mentioned above, and operation was decided for those patients.

Sugiyama et al. have reported that they detected adenoma or cancer at the rate of $14 \%$ in polyps with a size of 6-10 $\mathrm{mm}$ in their study (Goetze et al., 2010). Zielinski et al. have indicated that the risk of neoplasia increases in polyp size $\geq 6 \mathrm{~mm}$ and cholecystectomy should be applied to those patients (Zielinski et al., 2009).

Some limitations of our study should be accepted. Firstly, despite a great number of cases, it was a retrospective, single-center. Secondly, there were no patients in the adenoma-cancer group. Thirdly, US is a device-dependent examination and shows variability between doctors. Fourthly, some factors such as polyp's growth rate, body mass index, eating 
habits, family history, and occupations could not be analyzed.

\section{Conclusion}

Consequently, it is difficult to diagnose premalignant and malignant lesions and to differentiate them from benign lesions during preoperative period. The underlying transformation mechanism requires more molecular studies. We have the opinion that surgical treatment is the right approach for all GBP that are symptomatic, with accompanying stones, that have the ultrasonographic risk of malignancy.

\section{Acknowledgment}

All statistical analysis has been made by consulting to Prof. Dr. Erol Egrioglu (a member of Giresun University, Department of Statistics and research visitor in Lancaster University until September 2021) and he used SPSS package program licensed to Lancaster University, UK for all statistical analysis.

Ethics Committee Approval: For a retrospective study, permission was obtained from Giresun University Professor Doctor İlhan Özdemir Head Physician of Education and Research (Number: 30474902-100-E023783, Date: 02.06.2020).

Peer-review: Externally peer-reviewed.

Author Contributions: Concept- A.K.; DesignA.K., E.E.; Materials- A.K., E.E; Data Collection and Processing- A.K., E.E; Literature Review- A.K., E.E.; Writing- A.K., E.E.; Critical Review- A.K., E.E.

Conflict of Interest: No conflict of interest was declared by the author.

Financial Disclosure: The author declared that this study hasn't received no financial support.

\section{References}

Albores-Saavedra J, Chable-Montero F, GonzalezRomo MA, Jaramillo MR, Henson D. Adenomas of the gallbladder. Morphologic features, expression of gastric and intestinal mucins, and incidence of high-grade dysplasia/carcinoma in situ and invasive carcinoma. Hum Pathol 2012;43:1506-

Cairns V, Neal CP, Dennison AR,Garcea G. Risk and Cost-effectiveness of Surveillance Followed by Cholecystectomy for Gallbladder Polyps. Arch Surg 2012;147:1078-83.
Cha BH, Hwang J, Lee S, Kim J, Cho J, Kim H et al Pre-operative factors that can predict neoplastic polypoid lesions of the gallbladder. World $\mathrm{J}$ Gastroenterol. 2011;17:2216-2222

Csendes A, Burgos AM, Csendes P, Smok G, Rojas J. Latefollow-up of polypoid lesions of the gallbadder smaller than $10 \mathrm{~mm}$. Ann Surg 2001;234(5):657-60.

Dinc T, Coskun F, KutluKucuk O. Our surgical experience in gall bladder polyps. Gaziantep Med J 2013;19(3): 169-172

Goetze TO, Paolucci V. Adequate extent in radical reresection of incidental gallbladder carcinoma: analysis of the German Registry. SurgEndosc. 2010 Sep;24:2156-64.

Jones-Monahan KS, Gruenberg JC, Finger JE, TongGK. Isolated small gallbladder polyps: an indication forcholecystectomy in symptomatic patients. Am Surg2000; 66: 716-9.

Kwon W, Jang JY, Lee SE, Hwang DW, Kim SW. Clinicopathologic features of polypoid lesions of the gallbladder and risk factors of gallbladder cancer. J Korean Med Sci. 2009 Jun;24:481-7.

Lee KF, Wong J, Li JC, Lai PB. Polypoid lesions of thegallbladder. Am J Surg 2004;188(2):186-90.

Lin WR, Lin D-Y, Tai D-I, Hsieh S-Y, Lin C-Y, Sheen I-S et alPrevalence of and risk factors for gallbladder polyps detected by ultrasonography among healthy Chinese: analysis of 34669 cases. J GastroenterolHepatol 2008;23:965-9.

Lou MW, Hu WD, Fan Y, Chen JH, E ZS, Yang GF. CT biliarycystoscopy of gallbladder polyps. World J Gastroenterol2004;10(8):1204-7.

Marangoni G, Hakeem A, Toogood GJ, Lodge P, Prasad R. Treatment and surveillance of polypoid lesions of the gallbladder in the United Kingdom. HPB (Oxford) 2012; 14:435-40.

Sarkut P, Kilicturgay S, Ozer A, Ozturk E, Yilmazlar T. "Gallbladder polyps: factors affecting surgical decision." World Journal of Gastroenterology: WJG 19.28 (2013): 4526.

Shinkai H, Kimura W, MutoT. Surgical indications for smallpolypoid lesions of the gallbladder. Am J Surg 1998;175(2):114-7.

Sun XJ, Shi JS, Han Y. Diagnosis and treatment of polypoidlesions of the gallbladder: report of 194 cases. HepatobiliaryPancreat Dis Int 2004;3(4):591-4.

Yongliang S, Yang Z, Lan X, TanH "Neoplastic polyps in gallbladder: a retrospective study to determine risk factors and treatment strategy for gallbladder polyps." Hepatobiliary Surgery and Nutrition8.3 (2019): 219. 
Xu Q, Tao L-Y, Wu Q, Gao F, Zhang F-L, Yuan L, et alPrevalences of and risk factors for biliary stones and gallbladder polyps in a large Chinese population. HPB (Oxford) 2012;14:373-81.

Yildirim M, Erkan N, Yakan S, Boz A, Vardar E. Polyps of the Gallbladder: Retrospective Analysis of 33 Cases. Journal of ADÜ Faculty of Medicine. 2005; 6(1) : 27 - 30.

Yuksel A,Coskun M, Turgut HM, Ozyıldız M, Yazıcioglu MB, YildizSY. Postoperative Histopathology Findings of Ultrasonographically diagnosed Gallbladder Polyp.Kocaeli Medical Journal 2016; 5;1:11-15

Zielinski MD, Atwell TD, Davis PW, Kendrick ML, Que FG. Comparison of surgically resected polypoid lesions of the gallbladder to their preoperative ultrasound characteristics. J Gastrointest Surg. 2009;13:19-25. 